\title{
Inteligência e conhecimento específico em jovens futebolistas de diferentes níveis competitivos
}

\author{
J.C. Costa \\ J. Garganta \\ A. Fonseca \\ M. Botelho
}

\author{
Faculdade de Ciências do Desporto e de Educação Física \\ Universidade do Porto \\ Portugal
}

\begin{abstract}
RESUMO
A presente investigação visa avaliar e comparar a inteligência geral e o conhecimento específico do jogo em jovens praticantes de Futebol segundo o nível competitivo.

Para tal, foram utilizados o teste de atenção de Toulouse-Piéron, o teste das figuras idênticas de Thurstone e as matrizes progressivas de Raven, para avaliar os processos cognitivos gerais dos jovens futebolistas, bem como o protocolo de avaliação do conhecimento específico do jogo construído por Mangas (1999) e aperfeiçoado por Correia (2000). Estes instrumentos foram aplicados a uma amostra de 44 praticantes federados de Futebol de diferentes níveis competitivos, com uma média de idades de $16.00 \pm 0.53$ para o grupo de nível competitivo superior e de $16.13 \pm 0.63$ para o grupo de nível competitivo inferior. Os resultados mostraram uma tendência de maior conhecimento específico da modalidade em situações ofensivas para o grupo de futebolistas federados de nível competitivo superior, em relação aos de nível competitivo inferior, apesar das diferenças encontradas não se terem revelado estatisticamente significativas. Inversamente ao sucedido para o conhecimento específico do jogo, no que respeita à inteligência geral, os jogadores com nível competitivo inferior apresentaram valores superiores e estatisticamente significativos quando comparados com os do grupo de nível competitivo superior.
\end{abstract}

Palavras-chave: Futebol; Táctica; Inteligência; Conhecimento específico do jogo.

\author{
ABSTRACT \\ Intelligence and game understanding in young soccer players \\ at different competitive levels
}

In this research, it is intended to evaluate and to compare the general intelligence and the specific knowledge of the game in young Soccer practitioners according to the competition level.

The Toulouse-Piéron attention test, the Thurstone identical figures test and the Raven Progressive Matrixes (to evaluate the general cognitive processes of the young Soccer players) were used to evaluate the general cognitive processes of the young soccer players. To evaluate the game's specific knowledge a protocol built by Mangas (1999) and improved by Correia (2000) was used. These instruments were applied to a sample of 44 Soccer federate practitioners of different competition levels, with an age average of $16.00 \pm 0.53$ for the higher competition level group and $16.13 \pm 0.63$ for the lower competition level group.

The group of federate Soccer players of high competition level showed a greater specific knowledge of the game in offensive situations than those of lower competition level, in spite of the differences found haven't revealed themselves as statistically significant. Inversely to what occurred with the specific knowledge of the game, with the general intelligence the players with a lower competition level showed statistically higher results when compared to those of the higher competition level group.

Keywords: Soccer; Tactics; Intelligence; Game Understanding. 


\section{INTRODUÇÃO}

Apesar da manifesta dificuldade em definir com precisão as variáveis mais importantes a estudar para conhecer as razões conducentes ao sucesso $(11,60)$, a investigação em ciências do desporto tem apontado o desenvolvimento das habilidades perceptivas e cognitivas como um dos fundamentos e requisitos essenciais para a excelência da performance desportiva $(53,44,102,73,89,78,95,115)$.

No sentido de apurar como se formam os expertos no desporto, tem aumentado o número de estudos onde são analisadas as suas qualidades perceptivas e as componentes estratégicas, quer por indagação retrospectiva, quer por testes de conhecimento declarativo e processual, reconhecimento de padrões de jogo, protocolos verbais para análise das estratégias de tomada de decisão, análise dos comportamentos visuais dos desportistas expertos e não expertos (114).

Perante a, aparente, necessidade dum conhecimento sustentado e da existência de processos de recolha e utilização deste conhecimento, importa saber que tipo de relações existem, de facto, entre o conhecimento e o processamento geral. Mais concretamente, será que os jogadores que possuem maior inteligência geral evidenciarão um maior nível de conhecimento específico da sua modalidade? E relativamente à performance desportiva, poderá um melhor conhecimento do jogo contribuir, de alguma forma, para um nível de rendimento superior?

\section{OBJECTIVO DO ESTUDO}

Com o presente estudo pretendemos analisar e comparar a forma como a inteligência geral (IG) dos jogadores de Futebol e o seu conhecimento especifico do jogo (CE) se relacionam e se complementam, considerando diferentes níveis competitivos.

\section{REVISÃO DA LITERATURA}

\subsection{Natureza contextual do jogo de Futebol}

Os Jogos Desportivos Colectivos (JDC) caracterizam-se pela aciclicidade técnica, solicitações morfológico-funcionais diversas e intensa actividade psíquica, num contexto permanentemente variável, de cooperação-oposição $(94,49,40,45,69,83)$, com uma importância capital dos aspectos estratégico-tácticos
(37), onde a tomada de decisão precede a execução $(18,50)$.

Os inúmeros problemas surgidos no jogo obrigam o jogador a decidir e seleccionar qual a solução mais consentânea com a situação, obedecendo aos princípios gerais do jogo através do conhecimento que possui do jogo. Os jogadores têm que saber o que fazer, para depois seleccionar como o fazer, utilizando a acção motora mais adequada ao problema (6, 40). Assim, sem conhecer a essência do jogo e dos seus princípios tácticos, não se pode aproveitar na plenitude os recursos técnicos (75)).

O Futebol é considerado o JDC mais imprevisível e aleatório (29), características que resultam do envolvimento aberto (51), do elevado número de jogadores e da dimensão do espaço de jogo, bem como da duração do tempo de jogo. Neste sentido o jogo de Futebol reclama dos praticantes uma elevada capacidade perceptiva (40) e maiores exigências relativamente à componente visual que os restantes JDC (21).

Ao jogador é exigido que decida e exteriorize o seu raciocino mental ou decisão cognitiva (89). De todas as actividades desportivas é nos JDC que a táctica adquire o seu mais alto nível de expressão (44). Deste modo, diversos autores $(107,106,89,44,12$, 37) salientam a importância do desenvolvimento da táctica, pois nos JDC o principal problema que se coloca aos indivíduos que jogam é essencialmente táctico.

Concordamos assim com Garganta \& Cunha e Silva (41), quando defendem que: "Cada sujeito percebe o jogo, as suas configurações, em função das aquisições anteriores e do estado presente. Perante o fenómeno jogo, o observador constrói uma paisagem de observação, entendida como um conjunto de estímulos organizados face ao ponto de vista que ele possui sobre o fenómeno." (p.5).

O sucesso nos jogos tácticos depende largamente do nível de desenvolvimento das faculdades perceptivas e intelectuais dos atletas, especialmente em associação com outros factores que determinam a performance (88).

\subsection{Cognição: a faceta não visivel do processo táctico- -estratégico}

As acções tácticas distinguem-se das restantes acções desportivas, porque o seu desenvolvimento 
dá lugar a processos intelectuais muito mais alargados e qualitativamente superiores. O acto táctico é, na sua essência, a aplicação criadora do saber, das diferentes capacidades e do "savoir faire" (57). A determinação táctica do movimento desportivo pressupõe uma actividade cognitiva $(42,89,8,23$, $21)$, todavia inadequada e insuficientemente trabalhada $(52,94,98)$.

Em cada acção o jogador avalia as possibilidades de êxito e prepara mentalmente a acção a realizar em função da antecipação do comportamento dos adversários e da acção que os companheiros prevêem realizar-se nesse contexto (43), exigindo-se-lhe decisões inteligentes através de processos cognitivos (21).

De acordo com Rink, French \& Tjeerdsma (78) e Garganta $(37,39)$, a investigação permite identificar um conjunto de traços cognitivos e motores característicos do jogador experiente nos jogos desportivos. Constata-se assim a presença, a nível cognitivo, de: (i) conhecimento declarativo e processual mais organizado e estruturado; (ii) processo de captação de informação mais eficiente; (iii) processo decisional mais rápido e preciso; (iv) mais rápido e preciso reconhecimento dos padrões de jogo (sinais pertinentes); (v) superior conhecimento táctico; (vi) uma maior capacidade de antecipação dos eventos do jogo e das respostas do oponente; e (vii) superior conhecimento das probabilidades situacionais (evolução do jogo).

Ao nível da execução motora evidenciam: (i) elevada taxa de sucesso na execução das técnicas durante o jogo; (ii) maior consistência e adaptabilidade nos padrões de movimento; (iii) movimentos automatizados, executados com superior economia de esforço; e (iv) superior capacidade de detecção de erros e de correcção de execução.

Para Konzag (52), os desportistas com um elevado nível de desempenho em situação de jogo, possuem processos cognitivos de alto nível, nos quais a recepção e elaboração das informações são mais rápidas e mais precisas.

\subsection{Estrutura do comportamento táctico inteligente em futebol}

É através dum complexo mecanismo que engloba a percepção e análise da situação, a decisão a tomar e a execução $(57,1,98,36)$ que a táctica se consubstancia como elemento central e coordenador dos jogos de oposição $(2,6,44,1,77,89,71)$. O sistema de referências múltiplas em que os atletas actuam sugere uma implicação específica das funções psicológicas, nomeadamente no que se reporta aos aspectos cognitivos.

\subsubsection{Inteligência geral}

A capacidade de inteligência tem constituído uma das preocupações fundamentais dos psicólogos (31) e constitui um assunto de interesse de professores e gestores de recursos humanos (4), apesar de permanecer também como um conceito genérico, ambíguo e de difícil definição $(92,115)$.

Os autores estão, no entanto, de acordo que para compreender a inteligência é necessário identificar os processos que no seu conjunto constituem o comportamento inteligente (93).

Alguns autores comprovaram a importância da inteligência ao verificarem a existência de elevada correlação entre inteligência e a velocidade de processamento da informação (28) e ao encontrarem relação entre aprendizagem e inteligência (20).

Em suma, o conceito de inteligência é considerado vago, generalista e evasivo $(48,4)$. Reporta-se fundamentalmente a noções e conhecimentos académicos, dado o carácter abstracto de que os estudos habitualmente se revestem. Consiste predominantemente na exteriorização racional e teórica em detrimento de habilidades interventivas e práticas como as artes ou o desporto exigem, apesar de alguns autores apontarem cada vez mais inteligências específicas para determinados domínios.

A inteligência é assim entendida como uma confluência de requisitos e capacidades, tratando-se de um constructo, de uma conjugação de capacidades de compreensão, atenção, velocidade e memorização que relacionadas permitem ao sujeito desempenhar determinadas tarefas com maior precisão e acuidade. Os profissionais e investigadores do desporto questionaram-se inúmeras vezes sobre a relação entre os factores de inteligência estabelecidos pelos testes psicológicos e as habilidades técnicas e tácticas desportivas (13).

A inteligência geral parece ser um factor importante no processo de escolha e decisão desportivas, contu- 
do com uma relevância relativamente discreta, pois não parece explicar as diferenças individuais de performance dos jogadores $(19,86)$.

As investigações sobre a relação entre o êxito desportivo e o coeficiente de inteligência não têm obtido dados concretos. No entanto, algumas das aptidões assinaladas no domínio cognitivo, como a orientação espacial, têm um papel não negligenciável (104).

Esta temática vem sendo estudada por diversos autores. Botelho (14) num estudo com ginastas, concluiu que estes são mais rápidos e concentrados que indivíduos sedentários. Carvalho (22) no âmbito do Futebol, concluiu que os atletas de elite apresentaram níveis superiores de inteligência, assim como melhor velocidade atencional e velocidade perceptiva e, quanto à exactidão das respostas, o grupo amador apresentou resultados superiores ao de elite. Mendelsohn (65), num trabalho com jogadores de Futebol profissional, concluiu que o grau de concentração dos jogadores é proporcional ao grau de responsabilidade defensiva. Veiga, Colaço e Brito (112) estudaram a atenção e a inteligência geral (factor g) em ginastas, e concluíram que o sector masculino apresenta melhores índices de atenção, com uma significativa relação entre atenção e prestação desportiva, e registaram uma correlação significativa entre atenção e Inteligência.

\subsubsection{Conhecimento táctico}

A compreensão dos processos cognitivos utilizados pelos jogadores durante a competição é limitada. Ainda assim, é sabido que estes têm origem no conhecimento táctico, altamente especializado e armazenado na memória de longo prazo. A performance superior manifestada por jogadores expertos em situações específicas não se evidencia quando as tarefas são irrelevantes para o domínio de acção do jogador (61).

Assim, o indivíduo que sabe jogar é um indivíduo conhecedor, não no que diz respeito a conhecimentos académicos, mas no que se refere a convenções interactivas, nas quais saber fazer, e saber quando fazer, são o mesmo saber (81).

Uma vez que a táctica depende dos conhecimentos tácticos adquiridos e da forma como os utilizamos (36), vários autores têm relacionado a natureza do conhecimento dos praticantes com a sua performance desportiva quer em Portugal, quer no estrangeiro, como comprova o quadro 1.

Quadro 1 - Quadro resumo dos autores, métodos e amostra utilizadas em estudos no âmbito do conhecimento específico do jogo.

\begin{tabular}{|c|c|c|}
\hline Autor & Métodos & Amostra \\
\hline \multicolumn{3}{|l|}{ Portugal } \\
\hline Tavares \& Vicente (1991) & Sequências de imagens de jogo em vídeo e TV & $\begin{array}{l}28 \text { alunos de opção Basquetebol da FCDEF } \\
\text { (praticantes e não praticantes) }\end{array}$ \\
\hline Tavares (1993) & Sequências de imagens de jogo em PC & $\begin{array}{l}99 \text { Basquetebolistas, seniores }(24,7 \pm 4,4 \\
\text { anos de idade) e cadetes }(15,6 \pm 0,7 \text { anos } \\
\text { de idade })\end{array}$ \\
\hline Brito (1995) & $\begin{array}{l}\text { Sequências de imagens de jogo em vídeo } \\
\text { (resposta e tempo) }\end{array}$ & $\begin{array}{l}42 \text { Futebolistas: } 21 \text { Federados e } 21 \text { não } \\
\text { Federados; Juniores, juvenis e iniciados }\end{array}$ \\
\hline Machado (1996) & $\begin{array}{l}\text { Sequências de imagens de jogo em vídeo } \\
\text { (resposta e tempo) }\end{array}$ & $\begin{array}{l}40 \text { Futebolistas: } 20 \text { seniores ( } 24,4 \text { anos de } \\
\text { idade) e } 20 \text { iniciados ( } 13,7 \text { anos de idade) }\end{array}$ \\
\hline Tavares (1996) & Sequências de imagens de jogo em vídeo e TV & $\begin{array}{l}30 \text { Basquetebolistas, dois grupos (24,8 e } \\
15,3 \text { anos de idade) }\end{array}$ \\
\hline Dias et al. (1996) & $\begin{array}{l}\text { Sequências de imagens de jogo em PC } \\
\text { (resposta e tempo) }\end{array}$ & $\begin{array}{l}19 \text { Voleibolistas: } 9 \text { seniores }(25,3 \pm 3,28 \\
\text { anos de idade ) e } 10 \text { juvenis }(16,33 \pm 0,52 \\
\text { anos de idade) }\end{array}$ \\
\hline Pinto (1997) & $\begin{array}{l}\text { Questionário de escolha múltipla sobre regras } \\
\text { e princípios do jogo }\end{array}$ & $\begin{array}{l}120 \text { Basquetebolistas cadetes dos } 15 \text { aos } 18 \\
\text { anos de idade }\end{array}$ \\
\hline
\end{tabular}




\begin{tabular}{ll} 
Brito \& Maçãs (1998) & $\begin{array}{l}\text { Sequências de imagens de jogo em vídeo } \\
\text { (resposta e tempo) }\end{array}$ \\
Rodrigues (1998) & $\begin{array}{l}\text { Teste de sequências de imagens de jogo em vídeo } \\
\text { e teste teórico } \\
\text { Sequências de imagens de jogo em PC (resposta) }\end{array}$ \\
Mangas (1999) & $\begin{array}{l}\text { Sequências de imagens de jogo em PC } \\
\text { (resposta e tempo) }\end{array}$ \\
Correia (2000) & $\begin{array}{l}\text { Sequências de imagens de jogo em PC } \\
\text { (resposta e tempo) } \\
\text { Miragaia (2001) }\end{array}$ \\
Frazão (2001) & $\begin{array}{l}\text { Sequências de imagens de jogo em PC } \\
\text { (resposta e tempo) } \\
\text { Simulador computadorizado da competição de } \\
\text { orientação }\end{array}$ \\
\hline Brasil &
\end{tabular}

\section{Brasil}

Sisto \& Greco (1995) Esquemas de jogo para decidir e justificar qual a melhor solução

Greco et al. (1998) Sequências de imagens de jogo em Slides, fotos e vídeo

Paula (2000)

Espanha

Lumbreras [1991)

Safont-Tria et al. (1996)

Bayó \& Roca Balasch (1998)

Santesmases [1998]

\section{EUA}

McPherson \& French (1991) Sequências de imagens de jogo em PC (resposta e tempo)

Griffin et al. (1995)

McPherson (1999)

Bélgica

Helsen \& Pauwels (1987)
Validação de uma sequências de imagens de Voleibol para avaliar o CD e a TD

Análise ao vivo de trajectória inicial da bola, previsão do local em que esta toca no solo

Observação e análise do comportamento táctico e decisional

Testes de Inteligência desportiva em software próprio

Teste gráfico/esquema em desenho e em jogo
Entrevista e análise de conteúdo às soluções de jogo apresentadas em vídeo

Situações de jogo em vídeo projectadas em filme
21 Futebolistas federados: 7 sub-18, 7 sub-16 e 7 sub-14; e ainda 21 não federados 50 Basquetebolistas: com média de $16,5 \pm 0,7$ anos de idade

277 Futebolistas: 72 DE e 205 Federados, de 13 e 14 anos de idade 161 Futebolistas: com idades entre os 8 e os 18 anos de idade da AD Sanjoanense 40 Futebolistas Sub-17, de diferentes níveis competitivos

36 Futebolistas profissionais da I e II Liga porportuguesa e da $2^{\text {a }}$ Divisão $B$, entre 22 e 35 anos 30 praticantes de orientação divididos em três grupos de mestria

45 Andebolistas: dos 14 aos 18 anos de idade

614 sujeitos: 566 jogadores ( 155 voleibolistas, 252 andebolistas e 159 Futebolistas) e 48 treinadores

12 técnicos de voleibol de nível internacional

40 praticantes de ténis ( 20 de elite e 20 principiantes]

1 Futebolista durante 4 jogos

233 sujeitos: 60 alunos do INEF ( 18 a 31 anos de idade), 135 estudantes ( 12 a 17 anos de idade) e 38 de Escolas de Futebol ( 10 a 17 anos de idade)

83 Basquetebolistas dos 12 aos 19 anos de idade

17 estudantes ( 8 raparigas e 9 rapazes) com média de 22,4 anos de idade, praticantes de ténis

43 estudantes do $6^{\circ}$ ano: 22 grupo táctico e 22 técnico, no ensino do Voleibol 12 Tenistas ( 6 expertos e 6 principiantes)

20 Futebolistas de 21 anos de $8 \mathrm{~mm}$ numa parede, responde e executa a decisão táctica mais ajustada com bola 
Deste modo, são destacados pela literatura dois tipos de conhecimento relevantes para a performance desportiva: o conhecimento declarativo e o conhecimento processual $(4,37,43,58)$.

A especificidade desportiva implica um conhecimento das acções (conhecimentos declarativos) e conhecer como utilizar as acções (conhecimento processual) (9). Estes dois tipos de conhecimento diferenciam-se, sendo o "conhecer que" ou conhecimento declarativo e o "conhecer como" ou conhecimento processual.

Segundo Thomas, French \& Humphriest (105), o conhecimento declarativo pode ser definido como o conhecimento do regulamento da modalidade, aspectos das posições dos jogadores, estratégias básicas de defesa e ataque, enquanto o processual se refere às acções no decorrer do jogo.

Por outro lado, o conhecimento processual constitui a realização dum comportamento que dificilmente explicamos (43).

A metodologia de avaliação mais utilizada nos últimos tempos para o estudo desta problemática tem-se baseado em testes que apresentam situações de jogo, em vídeo ou filme, nos quais, após o visionamento, se decide qual a acção táctica mais ajustada (96). Méndez Giménez (68), numa revisão da literatura pelos diversos trabalhos em diferentes modalidades, comparando o nível de conhecimento de jogo (declarativo e processual) dos praticantes, concluiu que os estudos analisados apontam o ensino voltado para os conceitos tácticos como potenciador do desenvolvimento do conhecimento declarativo $(34,63,110$, $109,111,35,67)$.

\subsubsection{Tomada de decisão táctica}

A tomada de decisão consiste na capacidade de tomar decisões rápidas e tacticamente exactas, constituindo uma das mais importantes capacidades do atleta (88). Ela determina muitas vezes o sucesso dos jogos técnico-tácticos e é frequentemente responsável pelas diferenças na performance individual $(17,54,79,100,88,94,59$,).

Dum conjunto de possibilidades em permanente mudança o jogador escolhe a solução mais adequada à situação presente $(97,108)$. Nos JDC, acção é sinónimo de tomada de decisão, porque cada situação requer uma nova solução. É talvez este contexto de incerteza que proporciona aos JD uma "magia" particular (99).

Ripoll (80) concluiu, numa revisão aos diversos trabalhos realizados, que os expertos utilizam regras heurísticas para lidar com a multidimensionalidade das condições pressionantes (um comportamento visual sintético), processam apenas a informação relevante e eliminam as pistas não relevantes. Alves \& Araújo (8) acrescentam que quanto mais complexas as decisões, mais os expertos se distinguem dos iniciados.

Em meio instável e inconstante o praticante esforça-se em extrair as constâncias e regularidades no conjunto das informações disponíveis. O desportista experiente utiliza duas grandes categorias de estratégias: (1) encontrar regularidades nas modificações do envolvimento, (2) na construção de um repertório de esquemas que permitem ler a situação actual e de antecipar, a curto prazo, os acontecimentos numa base de tomada de informação, não sobre as acções do adversário, mas sobre as suas. Assim, os principiantes activam um único programa motor para controlar o conjunto do movimento, enquanto que os experientes disponibilizam ao longo da acção dois sub-programas funcionalmente independentes (30). A qualidade de decisão do atleta depende ainda, segundo Alves \& Araújo (8), do seu conhecimento declarativo e conhecimento processual específicos, das suas capacidades cognitivas e da competência da sua utilização.

\subsection{Inteligência vs conhecimento}

Inteligência e conhecimento parecem dois campos de intervenção diferentes mas inter-relacionados na procura de soluções e respostas para as dificuldades com que o jogador se depara. Existe, à primeira vista, uma espécie de descontinuidade, de ruptura, entre eles, pois têm sido abordados e confrontados em diferentes momentos, em distintos lugares e com diferentes preocupações e exigências teóricas diferentes. Mas, não é nosso propósito estabelecer e confrontar diferenças históricas, mas sim tentar esclarecer, a partir da sua especificidade relativa, que lugar poderá ocupar cada uma destas dimensões num campo único como o desporto. Podemos distinguir la memoria inmediata, la diferida y muchas otras formas de memoria; hay tantas 
como órganos sensoriales (memoria visual, auditiva, táctil...). Podemos distinguir la memoria inmediata, la diferida y muchas otras formas de memoria; hay tantas como órganos sensoriales (memoria visual, auditiva, táctil...). Podemos distinguir la memoria inmediata, la diferida y muchas otras formas de memoria; hay tantas como órganos sensoriales (memoria visual, auditiva, táctil...). Podemos distinguir la memoria inmediata, la diferida y muchas otras formas de memoria; hay tantas como órganos sensoriales (memoria visual, auditiva, táctil...). Podemos distinguir la memoria inmediata, la diferida y muchas otras formas de memoria; hay tantas como órganos sensoriales (memoria visual, auditiva, táctil...).

Como destacam Chi \& Glaser (24), o conhecimento específico de uma determinada área tem importância decisiva na solução efectiva dos problemas que essa área de actividade apresenta, razão pela qual alguns estudos recentes têm incidido nas interacções entre inteligência e conhecimento (4).

No presente estudo procuramos indagar se os jogadores que possuem maior inteligência geral evidenciam, também, um maior nível de conhecimento específico da sua modalidade, considerando os respectivos níveis competitivos.

\section{MATERIAL E MÉTODOS}

\subsection{Caracterização da amostra}

A amostra utilizada abrange 44 praticantes de Futebol federados (Sub-16 e Sub-17) do sexo masculino. Por exigência qualitativa dos objectivos do estudo, seleccionámos dois grupos de praticantes em campeonatos de níveis competitivos diferentes: 22 elementos participantes no Campeonato Nacional de Juniores B, com um nível competitivo superior (Grupo sup), com uma média de idades de 16 $\pm 0,52$, com uma prática desportiva federada média de $6,14 \pm 2,07$ anos e um total de 6 horas de treino semanal; e 22 elementos participantes no Campeonato Distrital da A. F. Porto, constituindo o grupo de nível competitivo inferior (Grupo inf), com uma média de idades de $16,1 \pm 0,62$, com uma prática desportiva federada média de $5,73 \pm 2,4$ anos e um total de 4,5 horas de treino semanal. As equipas seleccionadas pertencem à mesma área geográfica. As horas de treino semanal constituem a única característica geral em que se verifica a existência de diferenças estatisticamente significativas entre eles.

\subsection{Instrumentos de avaliação}

Face aos instrumentos de avaliação disponíveis para cada um dos parâmetros em estudo, seleccionámos e utilizámos aqueles que nos garantiam maior fiabilidade, validade e exequibilidade. Deste modo foram utilizados os seguintes meios para a avaliação dos jogadores:

\subsubsection{Protocolo de avaliação do conhecimento específico} do jogo A avaliação do conhecimento declarativo no âmbito dos JDC tem-se socorrido de protocolos de análise de prontidão e qualidade de resposta. Utilizámos um protocolo de avaliação do conhecimento construído e validado por Mangas (58), melhorado posteriormente por Correia (25) com a incorporação do factor tempo. O teste, composto por 11 imagens, foi classificado de acordo com o número de respostas correctas e respostas erradas de cada praticante, e ainda quanto ao tempo total de resposta às situações apresentadas.

\subsubsection{Testes de inteligência geral}

Face aos objectivos do nosso estudo seleccionámos algumas provas de natureza psicológica que nos pareceram importantes a fim de caracterizar cada uma das variáveis em estudo. Deste modo, todos os sujeitos foram submetidos a três provas específicas no intuito de medirmos os seguintes factores: Matrizes Progressivas de Raven PM 38 - "factor g de inteligência";

Teste de Barragem de Toulouse-Piéron (TP) - atenção concentração;

Figuras Idênticas de Thurstone (FI) - velocidade perceptiva.

\subsection{Procedimentos estatísticos}

Os procedimentos estatísticos utilizados, para além da estatística descritiva, consistiram no t-teste e na Anova unidimensional, para comparar diferenças de médias entre os dois grupos considerados, tendo-se recorrido ao coeficiente de correlação de Spearman para análise das correlações para a totalidade da amostra. O nível de significância foi mantido em $5 \%$. 


\section{RESULTADOS E DISCUSSÃO}

5.1. Comparação das classificações da inteligência geral versus classificação protocolo de avaliação do conhecimento do jogo
Constatámos ao comparar os grupos que a tendência de classificação evidencia uma supremacia do grupo de nível competitivo inferior nos testes de inteligência geral e do grupo de nível competitivo superior nos testes de CE (Quadro 2).

Quadro 2 - Classificações relativas por grupos ( $G$ sup e Ginf) para a velocidade atencional (Atvel), exactidão ou capacidade de concentração (Atexact), velocidade perceptiva (Fi), decisão/identificação (Rav) e conhecimento específico do jogo.

\begin{tabular}{|c|c|c|c|c|c|c|c|}
\hline \multirow[b]{2}{*}{ Classificação } & \multicolumn{3}{|c|}{ Inteligência Geral } & \multicolumn{4}{|c|}{ Conhecimento específico do jogo } \\
\hline & Rav & $\mathrm{Fi}$ & Atvel & Atexact & Correctas & Erradas & Tempo \\
\hline Melhor & Ginf & Ginf & Ginf & Ginf & G sup & G sup & $G$ sup \\
\hline Pior & G sup & G sup & G sup & G sup & Ginf & Ginf & Ginf \\
\hline
\end{tabular}

Alves (6) defende que a inteligência está relacionada com a fase de identificação dos estímulos, que, através duma estratégia visual mais eficiente, irá reflectir-se numa comparação mais rápida dos estímulos apresentados com os memorizados noutras situações. Verificamos neste estudo que ambas as metodologias exigem percepção e identificação dos estímulos pertinentes, contudo apenas no CE estes foram concretos e específicos da actividade desportiva, logo apenas aqui os diferentes níveis de rendimento e experiência se diferenciaram de acordo com a sua natureza qualitativa.

Comparámos as classificações do protocolo de avaliação do CE com os teste de IG, com o intuito de verificar se estão, de alguma forma, correlacionadas, facto que não se verifica.

Quanto a saber se os jogadores mais inteligentes noutras áreas do conhecimento também o são no desporto (86), a resposta encontrada aponta para que tal não se verifique, até porque as correlações encontradas indicam em sentido contrário e quando positivas atingem valores muito baixos.

\subsection{Resultados dos testes de inteligência geral} Quando comparamos as médias dos resultados da velocidade atencional (Atvel), exactidão ou capacidade de concentração (Atexact), velocidade perceptiva (Fi) e decisão/identificação (Rav) para ambos os grupos (Quadro 3), verificamos uma superioridade do $\mathrm{G}$ inf em todos os itens analisados. Importa ressalvar que na Atexact quanto menor o valor melhor é o resultado. Apesar da existência das referidas diferenças, apenas no Raven estas são estatisticamente significativas $(\mathrm{p}=0,04)$.

Quadro 3 - Média (X) \pm desvio padrão (dp) para os valores da velocidade atencional (Atvel), exactidão ou capacidade de concentração (Atexact), velocidade perceptiva (Fi) e decisão/identificação (Rav).

\begin{tabular}{|c|c|c|c|c|c|}
\hline & & Rav & $\mathrm{Fi}$ & Atvel & Atexact \\
\hline Grupo sup (n=22) & $X \pm d p$ & $40,86 \pm 12,12^{*}$ & $23,45 \pm 5,44$ & $195,40 \pm 45,43$ & $19,68 \pm 15,87$ \\
\hline Grupo inf (n=22) & $X \pm d p$ & $47,36 \pm 08,75$ & $26,18 \pm 7,61$ & $209,00 \pm 51,09$ & $18,82 \pm 14,37$ \\
\hline
\end{tabular}

* diferenças estatisticamente significativas entre os grupos ( $p \leq 0,05)$. 
As diferenças geográficas, causa de possíveis diferenças sociais e culturais entre os sujeitos, foram esbatidas ao seleccionarmos a amostra da mesma zona (Grande Porto). Contudo, apesar desta preocupação, é frequente, pela sua maior capacidade de recrutamento, que equipas como as do $\mathrm{G}$ sup seleccionem para os seus quadros jogadores de zonas do país mais distantes, apesar de agora residentes na mesma região.

Outros estudos como os de Veiga, Colaço e Brito (112), encontraram correlações positivas entre inteligência (factor g) e atenção e uma relação igualmente positiva entre atenção e prestação desportiva. Também Carvalho (22), em estudo com futebolistas seniores, encontrou uma relação positiva entre os testes psicológicos e o nível de prestação desportiva de cada grupo.
Se atendermos às características dos jogadores experimentados, indicadas por Ruiz Pérez (84), constatamos que os resultados por nós encontrados estão de acordo com algumas das características apontadas como: serem expertos no seu desporto concreto e não demonstrarem a sua superioridade em testes gerais.

\subsection{Comparação dos resultados do teste de conheci- mento específico do jogo}

Comparados os dois grupos da amostra verificamos, através do Quadro 4, que ao contrário dos testes de inteligência, nos testes de conhecimento do jogo o grupo $\mathrm{G}$ sup apresenta valores médios superiores ao $G$ inf, quer quanto à qualidade das respostas, quer quanto ao tempo de resposta às mesmas.

Quadro 4 - Média $(X) \pm$ desvio padrão (dp) para os valores das respostas correctas e erradas e dos tempos de decisão total nos dois grupos da amostra.

\begin{tabular}{lcccc} 
& & Respostas Correctas & Respostas Erradas & Tempo de Decisão Total (Seg.) \\
\hline Grupo sup $(\mathrm{n}=22)$ & TOTAL $X \pm d p$ & 145 & 11 & 931,25 \\
& & $6,59 \pm 1,7$ & $0,50 \pm 0,67$ & $42,32 \pm 14,03$ \\
Grupo inf $(\mathrm{n}=22)$ & TOTAL $X \pm d p$ & 123 & 16 & 949,17 \\
& & $5,59 \pm 1,73$ & $0,72 \pm 0,70$ & $43,14 \pm 14,20$
\end{tabular}

Ao nível das respostas correctas verificamos que os jogadores do $G$ sup $(6,59 \pm 1,7)$ obtiveram em média um valor superior aos jogadores do $\mathrm{G}$ inf

$(5,59 \pm 1,73)$; relativamente ao número de respostas erradas, encontramos também uma supremacia do $G$ sup $(0,5 \pm 0,67)$ sobre o $G \inf (0,72 \pm 0,7)$. Analisado o tempo médio utilizado para responder às situações apresentadas no protocolo, constatámos que o G sup $(42,32 \pm 14,03)$ continua a superiorizar-se ao $G$ inf $(43,14 \pm 14,20)$; como nos itens anteriores não existem diferenças estatisticamente significativas.

Parece assim confirmarem-se os resultados de estudos já realizados, segundo os quais se pensa que as equipas classificadas nas melhores posições obtêm os melhores resultados nos testes de conhecimento táctico (43).

Estudada a correlação verificamos que entre as respostas erradas e correctas existe uma correlação negativa $(r=-0,454 ; p=0,002)$, entre as respostas correctas e o tempo de decisão existe uma correlação positiva $(r=0,387 ; p=0,009)$ e entre a respostas erradas e o tempo de decisão $(r=-0,132 ; p=0,394)$ verificamos uma correlação que apesar de negativa não é estatisticamente significativa. Isto é, existe uma tendência para que aqueles que mais acertam serem também os que menos erros cometem. Assim como para aqueles que mais acertam utilizarem também menos tempo para responder.

Comparados os nossos resultados com os dos estudos realizados por Mangas (58) e Correia (25), em estudos com metodologia idêntica, encontramos um valor médio do número de respostas correctas do $\mathrm{G}$ sup do nosso estudo $(6,59 \pm 1,7)$ superior ao do mesmo escalão do estudo de Correia (25), $(5,62 \pm 1,85)$. Relativamente a Mangas (58), em escalão etário mais baixo apesar de federado e com um 
nível competitivo considerado também elevado $(6,97 \pm 1,63)$, obtivemos para ambos os grupos da nossa amostra resultados inferiores. Importa referir que, no caso deste estudo, os sujeitos testados não tiveram pressão temporal para tomar a decisão, uma vez que o tempo de resposta não foi uma variável tida em consideração.

Quando comparamos o número médio de repostas erradas, verificamos que a nossa amostra volta a evidenciar melhores resultados $(0,5 \pm 0,67$ do $G$ sup $)$ comparativamente com os estudos de Mangas (58) $(0,64 \pm 0,78)$ e de Correia (25) $(0,81 \pm 0,81)$.

Os nossos resultados parecem corroborar os encontrados por Helsen \& Pauwels (47), que investigaram praticantes de Futebol de recreação e praticantes federados com dez anos de prática em competição; e são também concordantes com os referidos nos estudos de French \& Thomas (34) e McPherson \& Thomas (62), estes realizados com basquetebolistas de diferentes níveis de experiência.

Contudo, conforme sustentam Williams \& Davids (113), o CE pode ser mais um requisito do que uma aquisição prática através da exercitação. Em estudo com futebolistas experientes, concluíram que o conhecimento declarativo é um pressuposto importante dos jogadores de elite e não resultado de elevada experiência, ou exposição ao contexto desportivo. Os diversos estudos de Greco $(42,43,44)$ alertaram para este facto aquando da validação dos testes para avaliar o conhecimento táctico. Segundo o autor, o nível de conhecimento táctico superior apresentado pelos atletas de clube pode justificar-se pelas exigências competitivas, bem como pela importância da componente táctica no treino. Com uma amostra de jovens praticantes de Futsal, demonstra que os atletas federados apresentam um conhecimento táctico superior ao revelado pelos praticantes do desporto escolar. No nosso estudo, apesar da supremacia evidente do G sup, os anos de experiência de cada um dos grupos analisados é idêntico, isto é, possuem tempo de prática idêntico, o que traduz uma experiência quantitativa semelhante. Considerando que o nível competitivo superior, em que actua o $\mathrm{G}$ sup, é fomentador de melhor experiência, apesar de não existirem diferenças estatisticamente significativas nos anos de prática federada, de acordo com Williams \& Davids (113) o maior nível de conhecimento dos jogadores mais experientes deve-se também à prática desportiva e não apenas à instrução. Pelo que este estudo vem corroborar outros já realizados nesta área (47, $79,53,94,102,66,58,25)$, nos quais se sugere que a qualidade de decisão está correlacionada com a experiência e anos de prática.

Williams, Davids \& Williams (114) sustentam que os atletas mais experientes possuem um conhecimento específico da modalidade mais amplo, permitindo-lhes identificar e valorizar determinadas situações e/ou soluções.

Relativamente aos diversos estudos realizados em Portugal, os valores obtidos no âmbito do CE acompanham a tendência manifestada pela investigação internacional.

Assim, comparativamente com os resultados encontrados por Tavares (94), com jogadores de

Basquetebol onde os mais experientes se revelaram mais rápidos no processamento da informação e na capacidade de decisão, quando comparados com jogadores menos experientes, denotámos total encontro com os resultados obtidos por uma amostra de basquetebolistas masculinos de dois níveis de experiência.

Posteriormente, Pinto (73) num trabalho na mesma modalidade, percorrendo diferentes áreas do conhecimento, mostrou-nos que os resultados apontam o conhecimento do jogo como a variável de maior poder preditivo no valor dos sujeitos. 0 teste de conhecimento do jogo permitiu descrever o nível de competência dos sujeitos nessa matéria e evidenciou sensibilidade suficiente para discriminar grupos distintos. Ainda no contexto do Basquetebol, Rodrigues (82), utilizando o teste validado por Pinto (73), concluiu, à semelhança do nosso estudo, que os jogadores federados possuíam conhecimento declarativo superior quando comparados com os do desporto escolar, sendo as questões tácticas aquelas que mais os diferenciaram. Também no protocolo referente à tomada de decisão, os federados optaram por decisões tácticas mais adequadas do que os do desporto escolar. Em 1999, Mendes comprovou em estudo na mesma modalidade, mas com iniciados masculinos, que os atletas com quatro ou mais anos de prática apresentam um conhecimento superior aos atletas com inferior tempo de prática. Daí decorre o alerta feito anteriormente quanto à questão da qualidade da expe- 
riência de cada grupo. Ou seja, até que ponto o número de anos de prática reflecte um nível superior de experiência?

Mais concretamente no âmbito do Futebol, Brito \& Maçãs (16) procuraram averiguar as diferenças de percepção e decisão técnico-táctica dos jogadores, segundo as suas idades e níveis competitivos.

Comparativamente ao nosso estudo, os resultados corroboram os encontrados relativamente à qualidade da resposta, mas contrariam-nos no que respeita ao facto de os jogadores não federados encontrarem as respostas mais rapidamente.

Relativamente ao trabalho de Mangas (58), com atletas federados (Sub-14), concluiu-se que os jogadores federados possuíam conhecimento declarativo superior quando comparados com os do desporto escolar, reforçando a tese de que a experiência adquirida através dum quadro competitivo mais vasto e rico contribui para a melhoria do conhecimento.

Da análise dos diversos estudos, parece lícito concluir-se que os atletas de melhor nível possuem um conhecimento mais elevado do que os seus colegas de nível inferior, facto comprovado pelos nossos resultados.

Tais resultados advêm, não só das maiores exigências a que o $\mathrm{G}$ sup está sujeito no seu processo de formação, mas também do maior tempo de exposição à aprendizagem, uma vez que apresentam um número de horas de treino semanais superior e com diferença estatisticamente significativa para o $\mathrm{G}$ sup. Vários estudos $(34,3,114,103,73,82)$ demostraram a existência de um maior conhecimento específico da modalidade por parte dos atletas mais experientes, o que leva a que estes reconheçam mais facilmente os problemas e identifiquem melhor as soluções.

Como sustenta Garganta (38): "De facto, à luz das exigências do desporto actual, não basta chegar mais longe, nem saltar mais alto, nem ser mais forte, é preciso ser mais rápido, mais veloz. Mais rápido, não apenas a chegar ao local desejado, ou a realizar uma acção, mas também a pensar, a encontrar soluções, a perceber o erro, a descodificar os sinais do envolvimento. Em síntese, mais rápido e melhor, a perceber, a pensar e a agir." (p.6). Os jogadores devem ter condições para inventar novos jogos no decorrer do próprio jogo (41).

\section{CONCLUSÕES}

O conjunto de resultados deste estudo remetem-nos para as seguintes conclusões:

i. os jogadores do mesmo nível competitivo diferem, entre eles, nas classificações obtidas em cada uma das avaliações realizadas (inteligência geral e conhecimento específico do jogo), isto é, os que revelaram melhor conhecimento específico do jogo não são os que apresentam melhores resultados nos testes de inteligência geral.

ii. os jogadores de nível competitivo inferior prevalecem sobre os de nível competitivo superior na velocidade perceptiva e atencional, factor geral de inteligência, capacidade de concentração e exactidão; logo os indicadores psicológicos gerais não se revelaram bons indicadores da performance desportiva;

iii.os grupos em estudo diferem significativamente quanto aos resultados obtidos nos testes de inteligência geral, na avaliação do factor geral de inteligência, destacando-se neste sentido os sujeitos do grupo de nível competitivo inferior;

iv. os jogadores com maior experiência e nível competitivo mais elevado apresentam um superior conhecimento específico do jogo.

\author{
CORRESPONDÊNCIA \\ Júlio Garganta \\ Gabinete de Futebol \\ Faculdade de Ciências do Desporto \\ e de Educação Física \\ Rua Dr. Plácido Costa, 91 \\ 4200.450 Porto \\ Portugal \\ jgarganta@fcdef.up.pt
}




\section{REFERÊNCIAS BIBLIOGRÁFICAS}

1. Aguillà GL, Pereira, PE (1993). Observación y evaluación en los deportes de cooperación-oposición: en busca de sus aspectos distintivos. Apunts: Educació Física i Esportes 31: 86-105.

2. Aguillà GL, Pérez, G, Solanas A, Renom J (1990). Aproximación a una propuesta de aprendizaje de los elementos tácticos individuales en los deportes de equipo. Apunts: Educació Física i Esportes 24: 59 - 68.

3. Allard F (1993). Cognition, expertise and motor performance. In: Starkes J, Allard F (Ed.). Cognitive Issues. In Motor Expertise. Amsterdam: Elsevier Science, 2: 17-34.

4. Almeida L (1994). Inteligência: definição e medida. Aveiro: Cidine.

5. Alves J (1983). Perfil psicológico de uma equipa de jovens adolescentes. Ludens 8: 20-24.

6. Alves J (1990). Inteligência e velocidade de processamento da informação: Contributo para a identificação das fases de processamento da informação mais influenciada pela inteligência. Tese de Doutoramento. FMH-UTL. Lisboa.

7. Alves J (1995). Processamento da informação e Inteligência. Lisboa: Edições FMH-UTL.

8. Alves J, Araújo D (1996). Processamento de informação e tomada de decisão no desporto. In: Cruz JF (Ed.). Manual de Psicologia do Desporto. Braga: SHO Lda, 361-388.

9. Anderson (1990). Cognitive psychology and its implications. San Francisco: W.H. Freeman and Company.

10. Araújo J (1995). Manual do treinador do desporto profissional. Porto: Campo das Letras.

11. Bauer G, Ueberle H (1988). Fútbol: Factores de rendimiento, direccion de jogadores y del equipo. Barcelona: Martinez Roca Ed.

12. Bayer C (1994). O ensino dos desportos colectivos. Lisboa: Dinalivro.

13. Bayó JM, Roca Balasch J (1998). Una propuesta de test de inteligencia deportivo (TID). Apunts: Educació Física i Esportes 53: 75-82.

14. Botelho M (1998). A actividade gímnica e os factores de eficácia no processamento de informação visual. Tese de Doutoramento. FCDEF-UP.

15. Brito J (1995). A decisão técnico-táctica no jogador de Futebol. Estudo comparativo dos processos perceptivocognitivos inerentes à decisão técnico-táctica em sujeitos dos 12 aos 18 anos, federados em futebol. Monografia de Licenciatura. UTAD. Vila Real.

16. Brito J, Maçãs V (1998). A decisão técnico-táctica no jogador de Futebol. Horizonte 81: 12-16.

17. Bronner R (1982). Decision making under time pressure. Lexington Books.

18. Bunker DJ, Thorpe RD (1982). A model for the teaching of games in secondary schools. Bulletin of Physical Education 19(1): 5-8.

19. Buscà B, Riera J (1999). Orientación deportiva hacia actividades tácticas. Revista de Psicologia del Deporte Vol. 8 (2): 271-276.

20. Campione JC, Brown AL, Bryant NR (1992). As diferenças individuais na aprendizagem e memória. In: Sternberg $\mathrm{R}$ (Ed.). As capacidade intelectuais humanas. Porto Alegre: Editora Artes médicas, 118-143.

21. Cárdenas D (2000). El entrenamiento integrado de las habilidades visuales en la iniciación deportiva. Málaga: Ed. Aljibe.
22. Carvalho AC (2000). Estudo comparativo dos processos cognitivos em jogadores de futebol com diferentes níveis de prática. Monografia de Licenciatura. FCDEF-UP.

23. Castelo J (1998). Estudo do factor táctico desportico. Os factores do treino desportivo. In: Castelo J et al (Ed.). Metodologia do treino desportivo. Lisboa: Ed. FMH-UTL, 188-250.

24. Chi M, Glaser R (1992). A capacidade para a resolução de problemas. In: Sternberg R (Ed.). As capacidade intelectuais humanas. Porto Alegre: Ed. Artes médicas, 249-276.

25. Correia P (2000). A decisão táctico-técnica em Futebol. Estudo comparativo dos processos Perceptivo-cognitivos inerentes à decisão táctico-técnica em Futebolistas federados dos 8 aos 18 anos de diferentes estatutos posicionais e anos de prática. Monografia de Licenciatura. FCDEF-UP.

26. Costa J (2001). Inteligência geral e conhecimento específico no Futebol. Estudo comparativo entre a Inteligência geral e o conhecimento específico em jovens futebolistas federados de diferentes níveis competitivos. Dissertação de Mestrado. FCDEF-UP.

27. Dias C, Tavares F, Moutinho C (1996). Influência de indicadores da informação no estudo da relação entre a rapidez de decisão e a adequação da resposta com os indicadores pertinentes recolhidos pelo blocador central em voleibol. In: Pinto D, Moutinho C (Eds.). Estudos 1, 11-16. CEJD/FCDEF-UP.

28. Donders FC (1969). On the speed of mental processes. Acta Psychologica 30: 412-431.

29. Dufour W (1993). Computer-Assisted Scouting in soccer. In: Reilly T, Larsys J \& Stibbe A (Eds). Science and Football II. London: E \& FN Spon, 160-166.

30. Duran C, Lasierra A (1987). Estudio experimental sobre didáctica aplicada a la iniciación de los deportes colectivos. Revista de Investigación y Documentación sobre las Ciencias de la Educación Física y del Deporte 7: 91-128.

31. Ferreira V (1998). A observação qualitativa. Determinismo de variáveis de presságio na competência de observação de diagnóstico em tarefas desportivas características das actividades gímnicas. Tese de Doutoramento. FMH-UTL. Lisboa.

32. Fonseca V (1996). Aprender a aprender, a educabilidade cognitiva. Lisboa: Notícias Editorial.

33. Frazão F (2001). Conhecimento processual e tomada de decisão em orientação. Comparação de jovens com diferentes níveis de mestria utilizando um simulador computadorizado. Dissertação de Mestrado. FCDEF-UP.

34. French KE, Thomas JR (1987). The relation of Knowledge development to children's basketball performance. Journal of Sport Psychology 9: 15-32.

35. Gabriele, Maxwell (1995). Direct versus Indirect Methods of squash instruction. Research Quarterly for Exercice and Sport 66 (Suppl.): A-63.

36. García Ucha F (2001). Factores psicológicos implicados en la táctica del tenis de campo. Educación Física y Deportes. Revista Digital 30. (http://www.efdeportes.com/).

37. Garganta J (1997). Modelação táctica do jogo de futebol. Estudo da organização da fase ofensiva em equipas de alto rendimento. Tese de Doutoramento. FCDEF-UP.

38. Garganta J (1999). O desenvolvimento da velocidade nos jogos desportivos colectivos. R. Treino Desportivo 6 ( $3^{\mathrm{a}}$ Série): 6-13.

39. Garganta J (2000). O treino da táctica e da estratégia nos jogos desportivos. In: Garganta J (Ed.). Horizontes e órbi- 
tas no treino dos jogos desportivos. Porto: FCDEF-UP e CEJD: 21-36.

40. Garganta J, Pinto J (1994). O ensino do futebol. In: Graça A, Oliveira J (Eds.). O ensino dos Jogos Desportivos. Porto: CEJD/FCDEF-UP: 97-137.

41. Garganta J, Cunha e Silva P (2000). O jogo de futebol: entre o caos e a regra. Horizonte 91: 5-8.

42. Greco JP (1988). Hándbal. La formación de jogadores inteligentes. Stadium 128: 22-30.

43. Greco JP (1999). Cognição e ação. In: Novos conceitos em treinamento esportivo. Brasília: CENESP-UFMG e INDD.

44. Greco JP, Chagas M (1992). Considerações teóricas da tática nos jogos desportivos coletivos. Revista Paulista de Educação Física de S. Paulo 6 (2): 47-58.

45. Gréhaigne J, Godbout P (1995). Tactical Knowledge in team sports from a constructivist prespective. Quest 47: 490-505.

46. Griffin LL, Oslin JL, Mitchell SA (1995). Analysis of two instructional approaches to teaching net games. Research Quaterly for Exercice and Sport 66 (Suppl.): A-64.

47. Helsen W, Pauwels J (1987). The use of a Simulator in Evaluation and Training of Tactical Skills. In: Soccer. Instituut voor Lichamelijke Opleiding, K. U. Leuven, Tervuursevest 101, 3030 Heverlee, Belgium: 13-17.

48. Hernández Mendo A, Manzano SG, Arcas JF (2000). Inteligencia Emocional vs Inteligencia Social: datos para un estudio con deportistas. Educación Física y Deportes. Revista Digital 23.

(http://www.sportquest.com/revista/http://www.efdeportes.com/).

49. Hernandez Moreno J (1994). Fundamentos del deporte: Análisis de la estrutura del juego deportivo. Barcelona: Ed. INDE.

50. Hernandez Moreno J (1995). Aproximacion praxiologica al analisis de la estrutura del deporte. Curso de Jogos Desportivos Colectivos, FCDEF-UP.

51. Knapp B (1963). La habilidad en el deporte. Valladolid: Ed. Miñon.

52. Konzag G (1992). Conocer y jugar. El problema de la objectivación de los antecedentes cognoscitivos del ejercício en los juegos deportivos. Stadium 152: 27-34.

53. Konzag I (1990). Attivita cognitiva e formazione del giocatores. Rivista di Cultura Sportiva 20: 14-20.

54. Lacombe D, Sarrazin C, Alain C (1986). Decision making in sport: na information processing experimental paradigm. In: L.E. Unestahl (Eds.). Sport Psychology and practice Orebro. Sweden: Veje Publ, Inc.: 179-189.

55. Lumbreras JM (1991). Propuesta de test encaminado a evaluar la percepción de trayectórias aéreas de objectos. Apunts: Educació Física i Esportes 25: 59-66.

56. Machado A (1996). A decisão técnico-táctica em Futebol. Estudo comparativo da decisão técnico-táctica em indivíduos federados no escalão iniciados e sénior. Monografia de Licenciatura. UTAD. Vila Real.

57. Mahlo F (s/d). Acte tactique en Jeu. Paris: Vigot.

58. Mangas C (1999). Conhecimento declarativo no futebol. Dissertação de Mestrado. FCDEF-UP.

59. Manno R, Becarinni C, D’Ottavio S (1993). Futbol - La formacion del joven jugador. Desarrolo físico-motor y processo de preparación y formación del joven deportista : un enfoque lectivo (primeira parte). Stadium 160: 7-12.
60. Marques A (1993). Bases para a estruturação de um modelo de detecção e selecção de talentos desportivos em Portugal. Espaço 1: 47-58.

61. McPherson SL (1999). Tactical differences in problem representations and solutions in collegiate varsity and beginner female Tennis players. Research Quarterly and Sport vol. 7 n 4 : 369-384.

62. McPherson SL, Thomas J (1989). relation of Knowledge and performance in boys tennis: age and expertise. Journal of Experimental Child Psychologi 48: 190-211.

63. McPherson SL, French KE (1991). Changes in cognitive strategies and Motor Skill in tennis. Journal of Sport and Exercise Psychology 13: 26-41.

64. Meinel K, Schnabel G (1987). Teoría del movimiento. Síntesis de una teoría de la motricidad deportiva bajo el aspecto pedagógico. Buenos Aires: Stadium.

65. Mendelsohn D (2000). Test de Toulouse-Pieron aplicado a jugadores de fútbol profesional (Club El Porvenir). Educación Física y Deportes. Revista Digital 18. (http://www.efdeportes.com/).

66. Mendes L (1999). A defesa no processo de preparação desportiva em basquetebol. Tese de Mestrado. FCDEF-UP.

67. Méndez Giménez A (1998). Efectos de las técnicas de enseñanza en el rendimiento físico-deportivo y en la motivación durante la iniciación deportiva. Póster presentado en el VII Congreso Mundial de Deportes para Todos. COE. (Actas en prensa).

68. Méndez Giménez A (1999). Modelos de enseñanza deportiva. Analisis de dos decadas de investigacion. Educacion Física e Deportes. Revista Digital 13. (http://www.efdeportes.com/).

69. Mesquita I (1998). A instrução e a estruturação das tarefas no ensino no Voleibol. Tese de Doutoramento. FCDEF-UP.

70. Miragaia C (2001). Conhecimento declarativo e tomada de decisão em futebol. Estudo comparativo da exactidão e do tempo de resposta de futebolistas de equipas da I, II e $2^{\text {a }}$ divisão B. Dissertação de Mestrado. FCDEF-UP.

71. Moya FE (1996). El concepto de táctica individual en los deportes colectivos. Apunts: Educació Física i Esportes 51: 16-22.

72. Paula P (2000). Processo de validação de teste para avaliar a capacidade de decisão tática e o conhecimento declarativo no voleibol: situações de ataque de rede. Dissertação de Mestrado. EEF-UFMG. Belo Horizonte.

73. Pinto D (1995). Indicadores de performance em Basquetebol. estudo descritivo e preditivo em cadetes masculinos. Dissertação de Mestrado. FCDEF-UP.

74. Pinto D (1997). Construção de um teste para avaliar o conhecimento declarativo no jogo. In: Marques A, Prista A \& Júnior AF (Eds). Educação Física: contexto e inovação (vol. II). Maputo-Moçambique: 337-348.

75. Read R, Devis JD (1990). Enseñanza de los juegos deportivos: cambio de enfoque. Apunts: Educació Física i Esportes 22: 51-56.

76. Richardson K (1991). Understanding intelligence. Milton Keynes: Open University Press.

77. Riera J (1995). Estratégia, táctica y técnica desportiva. Apunts: Educació Física i Esportes 39: 45-56.

78. Rink JE, French KE, Tjeerdsma BL (1996). Foundations for the learning and instruction of sports and games. Journal of Teaching in Physical Education 15: 399-417. 
79. Ripoll H (1987). Interaction des opération cognitives et des mécanismes sensorimoteurs dans les activités physiques et sportives. In: Von Hofe A \& Simonnet R (Eds.). Recherches en Psychologi du Sport. Paris: EAP, 68-85.

80. Ripoll H (1991). The understanding-acting processing sport: the relationship between the semantic and sensoriomotor visual function. Internacional Journal of Sport Psychology 22 (3/4): 221-250.

81. Roca Balasch J (1998). El ajuste temporal: criterio de ejecución distintivo de la inteligencia deportiva. Apunts: Educació Física i Esportes 53: 10-17.

82. Rodrigues J (1998). A capacidade de decisão táctica e o conhecimento de jogo em jogadores juniores de basquetebol. Estudo comparativo entre basquetebolistas federados e do desporto escolar. Dissertação de Mestrado. FCDEF-UP.

83. Romero Cerezo C (2000). Hacia una concepción más integral del entrenamiento en el fútbol. Educación Física y Deportes. Revista Digital 19. (http://www.efdeportes.com/).

84. Ruiz Pérez LM (1999). Rendimiento deportivo, optimizacion y excelencia en el deporte. Revista de Psicologia del Deporte 8 (2): 235-248.

85. Safont-Tria BB, Riera J, Traver V, Nicolau JP (1996). Propuesta de análisis de la táctica individual ofensiva en el fútbol. Apunts: Educació Física i Esportes 43; 63-71.

86. Sampedro J (1999). Fundamentos de la táctica deportiva. Madrid: Gymnos Ed.

87. Santesmases JS (1998). Formación cognoscitiva y rendimiento táctico. Apunts: Educació Física i Esportes 53: 33-41.

88. Schellenberger H (1990). Psychology of team sports. Toronto: Sports Book Publisher.

89. Sisto FF, Greco JP (1995). Comportamento táctico nos esportes colectivos. Revista Paulista de Educação Física 9: 63-68.

90. Souza P, Greco JP (1999) Desenvolvimento da percepção no futsal. In: Greco P et al. (Eds.). Temas Atuais em Educação Física e Esportes III. Belo Horizonte: Editora Healthy.

91. Souza P, Costa VT, Greco JP (1999). Validação do teste de conhecimento táctico. Futsal. In: Actas do $1^{\circ}$ Congresso Internacional de Ciências do Desporto. 7 a 9 Outubro 1999, FCDEF-UP.

92. Sternberg R (1992). A Capacidade Intelectual Geral. In: Sternberg R (Ed.). As capacidades intelectuais humanas. Porto Alegre: Editora Artes médicas, 17-42.

93. Sternberg R, Salter W (1986). Conceptions of intellegence. In: Stermberg R (Ed.). Handbook of Human Intellegence. Cambridge: Cambridge University Press, 3-28.

94. Tavares F (1993). A capacidade de decisão táctica no jogador de basquetebol. Tese de Doutoramento. FCDEF-UP.

95. Tavares F (1996). Tomada de decisão no Basquetebol: Aplicação de um teste-vídeo computorizado para avaliação. In: Pinto D, Moutinho C (Eds). Estudos 1. CEJD/FCDEFUP: $57-58$.

96. Tavares $F$ (1997). A pesquisa visual no desporto; quadro conceptual e métodos de investigação. Horizonte 75: 24-28.

97. Tavares F, Vicente C (1991). Construção de um modelo selectivo de imagens vídeo para avaliar as capacidades de análise de situações técnico-tácticas de basquetebol: estudo discriminante. In: Bento J, Marques A (Eds). As Ciências do Desporto e a Prática Desportiva. Desporto de rendimento, desporto de recreação e tempos livres (vol II). Porto: FCDEF-UP, 157- 163.

98. Tavares F, Faria R (1996). A capacidade de jogo como prérequisito do rendimento para o jogo. In: Oliveira J, Tavares F (Eds). Estratégia e táctica nos jogos desportivos colectivos. Porto: CEJD/FCDEF-UP, 39-50.

99. Tavares G (1994). O papel das regras na definição das lógicas dos desportos colectivos. Horizonte 60: 207-210.

100. Temprado J (1989). Prise decision en sport: Modallités d'etudeset donnés actuelles. STAPS 10 (19): 53-67.

101. Temprado J (1991). Les aprrentissages décisionnels en EPS. In: Famose J, Fleurance Ph. \& Touchard Y (Eds.). Appretissage moteur: rôle des representations. Paris: Editions Revue EPS, 131-156.

102. Tenenbaum G, Bar-Eli M (1992). Decision making in sport: a cognitive perspective. In: Singer R, Murphy M, Tennant L (Eds.). Handbook of Research on Sports Psychology. New York: Macmillan Publishing Company, 171-192.

103. Thomas K (1994). The development of sport expertise: From leeds to MVP legend. Quest 46: 199-210.

104. Thomas R (1993). Les aptitudes. In: Famose JP (Ed.). Cognition et performance. Paris: Insep, 219-234.

105. Thomas J, French K, Humphries C (1986). Knowledge development and sport skills performance: Directions for motor behavior research. Journal of Sport Psychology 8: 259-272.

106. Thorpe R (1983). Understanding approach to the teaching of tennis. Bulletin of Physical Education 19 (1): 12-19.

107. Thorpe R, Bunker D (1982). From theory to practice: two examples of an understanding approach to the teaching of games. Bulletin of Physical Education 18, (1): 9-15.

108. Tiegel G, Greco JP, Souza P, Filho E (1999). Teoria da ação e futebol. Não publicado.

109. Turner AP (1993). A model for working with students with varying knowledge structures. Artigo apresentado na American Alliance for Health, Physical Education, Recreation and Dance. Conferencia Nacional Washington, DC.

110. Turner AP, Martinek TJ (1992). A comparative analysis ot two models for teaching games -technique approach and game-centered (tactical focus) approach. International Journal of Physical Education 29 (4): 15-31.

111. Turner AP, Martinek TJ (1995). Teaching for understanding: a model for improving decision making during game play. Quest 47(1): 44-63.

112. Veiga A, Colaço C, Brito A (1997). Ansiedade, atenção e inteligência (fagtor $\mathrm{g}$ ) na prestação desportiva em Ginástica Artística. In: Marques A, Prista A \& Junior A (Eds). Educação Física: Contexto e Inovação (Vol. II): 371-379.

113. Williams M, Davids K (1995). Declarative Knowledge in Sport: A By-Product of Experience or a Characteristic of Expertise. Journal of Sport \& Exercice Psychology 17: 258-275.

114. Williams M, Davids K, Williams J (1999). Visual Perception and Action in Sport. London: E \& FN Spon.

115. Williams AM, Reilly T (2000). Talent identification and development in soccer. Journal of Sport Sciences 18: 657-667. 